\title{
Exosomes as Liquid Biopsy Biomarkers in Diffuse Large B-cell Lymphoma (DLBCL)- Current State-of-the-Art and Unmet Clinical Needs
}

\author{
Kenneth Ofori ${ }^{1}$, Govind Bhagat ${ }^{2}$, and Alex Rai ${ }^{2}$ \\ ${ }^{1}$ Columbia University Irving Medical Center \\ ${ }^{2}$ Affiliation not available
}

May 26, 2020

\begin{abstract}
Diffuse Large B-Cell Lymphoma is the most common type of Non-Hodgkin's Lymphoma. The disease exhibits significant clinical and biologic heterogeneity. Treatment with standard first line therapy results in cure in about $60 \%$ of patients while $30-40 \%$ of patients either are refractory to therapy or relapse. Current prognostic scores and biomarkers are unable to accurately predict patients who would relapse or would have refractory disease. A part of the heterogeneity in the behavior of DLBCL is explained by the cell of origin of the tumor. Germinal center type (GCB) DLBCL which is derived from centroblasts are associated with better prognosis compared with activated B-cell type (ABC), which is derived from a B-cell committed to secretory differentiation. While the gold standard for cell of origin determination is gene expression profiling, immunohistochemical methods are routinely used because of more readily available fixed tissue and expertise. Immunohistochemical methods are however associated with a significant degree of discordance with GEP. Within the ABC and GCB types of DLBCL, subgroups of prognostic significance have been identified using various multiple approaches which do not inure themselves to routine practice partly because of limitation of diagnostic material or expertise. Exosomes are a class of membrane bound extracellular vesicles of endosomal origin, produced by multiple cell types. They are involved in intercellular communication and present in abundance in various bodily fluids. Exosomal cargo which includes nucleic acids and proteins can be analyzed, yielding diagnostic and prognostic information in management of DLBCL.
\end{abstract}

\section{Introduction}

Diffuse large B-Cell lymphoma (DLBCL) is the most common type of Non Hodgkin Lymphoma (NHL) constituting $30-40 \%$ of cases $(1,2,3)$. An estimated 27,650 new cases were diagnosed in 2016 with 25,380 of them being the not otherwise specified (NOS) variant (4). DLBCL shows a slight male predominance (55\%) and the median age at diagnosis is 64 years, but the disease affects all age groups and age of onset is earlier in African Americans $(5,6)$. The 5-year survival rate shows mild geographic variation, $62 \%$ in the US (7) and $55.4 \%$ in Europe (8).

DLBCL, despite its relative morphologic homogeneity, represents heterogeneous entities, with disparate biological and clinical manifestations. Outcome of disease to standard first line therapy, R-CHOP, consisting of rituximab, cyclophosphamide, doxorubicin, vincristine, and prednisone plus the monoclonal antibody rituximab, is variable. Twenty to fifty percent of patients are either refractory to therapy without remission or relapse after complete remission $(9,10,11)$

Relapsed disease is associated with a poor prognosis and patients with early disease relapse have a worse prognosis compared to those with late relapses (12). The mechanisms for relapse are purportedly different for these two groups (13). The prognosis for refractory patients, patients whose disease fails to go into 
remission or progress with induction therapy, is even more dismal compared to early and late relapse disease (14). While patients with relapse and refractory disease might have had need for more aggressive first line therapies, there are currently no biomarkers that adequately predict at diagnosis, the patients who are likely to be refractory to therapy, relapse after initial remission, or the time of relapse whether early or late. Similarly, once treatment commences or remission is achieved, there are no biomarkers that can accurately predict subsequent behavior of the disease. In addition, in refractory and relapsed disease, biomarkers capable of predicting response to second or additional lines of therapy remain elusive. Thus, there exists an urgent, unmet clinical need for biomarkers that address these challenges in management of DLBCL.

Current disease stratification systems/methods and assays

International Prognostic Index for Risk Stratification of DLBCL patients

The International Prognostic Index (IPI) and its revised variant, R-IPI are widely used clinical prognostication systems for DLBCL $(15,16)$. These indices use a combination of age, Eastern Cooperative Oncology Group (ECOG) performance status, tumor stage, lactate dehydrogenase level, and the number of extra nodal sites of disease to predict the prognosis of patients with DLBCL (17). Biccler et al showed the poor performance of the IPI in predicting overall survival in DLBCL patients. (18) In a study investigating the ability of the IPI and the revised index to predict outcome, R-IPI identified three distinct prognostic groups with: (a) very good (4-year progression-free survival [PFS] 94\%, overall survival [OS] 94\%), (b) good (4-year PFS 80\%, OS 79\%), and (c) poor (4-year PFS 53\%, OS 55\%) outcomes, respectively $(\mathrm{P}<0.001)(19)$. However, neither the R-IPI nor IPI are unable to predict patients with $<50 \%$ chance of survival. Such patients potentially need more potent first lines of therapy. However, there are no biomarkers that can reliably identify these high risk patients as of yet.

\section{Cell of Origin (COO) determination}

The cell of origin of DLBCL explains part of the heterogeneity of the disease. Using gene expression profiling, DLBCL NOS was initially classified into germinal center B cell (GCB), activated B-cell (ABC), and nonclassifiable types (20). GCB type DLBCL is thought to derive from centroblasts while the ABC type has features reminiscent of a B-cell committed to terminal B-cell differentiation (21) (see Figure 1).

The COO classification could predict overall survival of DLBCL patients and response to R-CHOP therapy. The GCB subtype has a more favorable prognosis compared to the ABC subtype. (22,23,24,25). Determination of cell of origin also has implications on drug therapy in relapsed/refractory disease. Recently, ibrutinib, a BCR inhibitor was found to be effective in relapsed and refractory ABC type DLBCL (26).

The gold standard method for determining COO has been gene expression profiling (GEP) (27). This modality, however, is not available at all centers and many assays require fresh tissue, which may not always be available. In routine practice, various immunohistochemistry (IHC) based algorithms (see Figure 2) such as Hans, Tally, Choi and Visco-Young are used to determine COO for DLBCL $(28,29,30,31)$. IHC methods for COO assignment are more practical and widely available, but are plagued by inter-observer variability that can result in discordant classification compared with the gold standard. Gutiérrez-García et al demonstrated that when compared with GEP, different IHC algorithms; Colomo, Hans, Muris, Choi, and Tally, misclassified cases at a higher rate when defining the GCB subset: $41 \%, 48 \%, 30 \%, 60 \%$, and $40 \%$, respectively (32). Geneexpression profiling and not immunophenotypic algorithms predicts prognosis in patients with diffuse large B-cell lymphoma treated with immunochemotherapy. In this study, while the GEP-defined groups showed significantly different 5 -year progression-free survival ( $76 \%$ vs $31 \%$ for GCB- and ABC- DLBCL) and overall survival ( $80 \%$ vs $45 \%$ ), none of the IHC algorithms retained the prognostic impact of the COO groups (GCB vs non-GCB). Other studies have also suggested that IHC algorithms may not have the same prognostic impact as other methods of $\mathrm{COO}$ determination $(33,34,35)$. These results underscore the unmet clinical need for a methodology that uses readily available biopsy material for accurate COO classification, concordant with GEP while maintaining prognostic utility.

The COO classification is not without limitations however. COO does not explain all the heterogeneity in 
the behavior and prognosis of DLBCL. Using various approaches, subgroups within ABC showing favorable prognosis have been identified and adverse prognostic groups have also been identified for the GCB type. In a study involving 574 DLBCL biopsy samples using exome and transcriptome sequencing, array-based DNA copy-number analysis, and targeted amplicon resequencing of 372 genes to identify genes with recurrent aberrations, Schmitz et al. identified four DLBCL genetic subtypes: BN2, EZB, N1, and MCD, with the former two determined to have favorable prognosis and the latter two associated with poor prognosis (36). Progression free survival and overall survival varied significantly within these groups. Of note, heterogeneity in behavior within the $\mathrm{ABC}$ group could be identified, with inferior survival in the MCD and N1 subtypes of ABC and favorable survival in the BN2 subtype. In GCBs, EZB subtype had a worse predicted 5-year survival compared with other GCBs non-classifiable by this system. Significant independent and additive contributions to survival by gene expression profiling (ABC vs GCB) were noted in this genetic subtyping model.

Chapuy et al used a combination of recurrent mutations, somatic copy number alterations

and structural variants (SV) to identify five distinct groups of DLBCL including a hitherto unappreciated group of ABC-DLBCL with favorable prognosis (37). They also identified a subtype of GCB-DLBCL with poor prognosis characterized by mutations and structural variants of $B C L 2$, mutations in PTEN and chromatin modifiers such asKMT2D , CREBBP , and EZH2 and focal10q23.31/PTEN loss. These subgroups within the different $\mathrm{COO}$ types provide insight not only for prognosis but also suggest possible mechanisms for therapeutic intervention. In spite of the usefulness of these multiparametric approaches for classification, it will be challenging to apply these genetic classification models in the clinical settings due to limitations in the amount of diagnostic material available, resources and bioinformatics expertise.

Disease Monitoring During Therapy

The rationale of using multiple agent chemotherapies in the treatment of cancers is to avoid development of resistance, but resistance does develop regardless of the type and combination of therapy. The neoplastic cells may develop adaptive resistance to chemotherapy in the course of treatment. Serial tumor profiling can provide insight into the pathways mediating adaptive resistance and identify targets for novel therapeutic drug development to overcome the resistance mechanisms (38). Real time analysis may also allow prompt detection of resistance and suggest alteration of therapy if signatures of resistance portending refractoriness or relapse are detected. In DLBCL patients, it is not usually feasible to obtain tissue regularly for such profiling without invasive measures, which cause patient discomfort and increase the risk of infections from multiple biopsy procedures. Thus, a non-invasive method for tissue sampling at multiple time points would be of great benefit.

\section{Post Treatment Surveillance}

The role of post-therapy or remission imaging surveillance in the management of DLBCL patients is controversial. While the National Comprehensive Cancer Network (NCCN) and European Society of Medical Oncology (ESMO) guidelines recommend surveillance imaging for stage III/IV disease for the first two years after completion of front-line therapy (39), the 2014 Lugano classification system advises against routine surveillance imaging (40). The positive predictive value of post treatment PET is low, resulting in patient anxiety and increased costs for these unnecessary medical procedures $(41,42)$. Studies have also failed to establish a survival advantage in imaging detected disease relapse $(43,44)$. Realization of the lack of survival benefit of surveillance imaging has resulted in decreased rates of surveillance, although this practice is still quite frequent, with over half of DLBCL patients diagnosed in 2014-2016 undergoing surveillance imaging (45). Thus, there is a pressing need for more specific and sensitive methods for detecting recurrence of disease while also avoiding risks of radiation exposure (46).

Novel Methodologies in DLBCL Management- Liquid Biopsy Based Approaches

Liquid biopsy techniques which involve assessment of cancer related biomarkers in bodily fluid samples, have potential to resolve the unmet clinical needs in management of DLBCL. Common liquid biopsy techniques 
include circulating tumor cells (CTC), circulating tumor DNA (ctDNA) and exosomes.

Circulating Tumor Cells

Circulating tumor cells are neoplastic cells that are passively shed or actively intravasate into circulation from primary tumors and/or metastatic deposits. (47). The number of CTCs in cancer patients correlate with treatment outcome and overall survival (48). The DNA, RNA and protein content of CTCs can be evaluated for molecular characterization informative of native tumor tissue (49). CTCs constitute a small fraction of cells in circulation- 1 in $10^{9}$ cells (50) and thus various techniques using distinct biological and physical properties of CTCs are used to differentiate and isolate them from leucocytes, erythrocytes and other cells in a blood sample. The predominant method of CTC isolation involves enrichment for cells with EPCAM expression and lack of CD45 expression $(51,52,53)$. This is not applicable to circulating DLBCL cells and cells from other hematological malignancies which express CD45 and lack EpCAM expression. In addition, the low abundance of CTCs makes their use in early stage of disease challenging. Other challenges with use of CTC as liquid biopsy is the need for prompt processing of whole blood after collection in most isolation platforms (54) precluding the potential for biobanking specimens for future analyses or use of archival biobanked specimens for studies.

\section{Cell free DNA and Cell tumor DNA}

Non cell bound DNA fragments are shed into circulation as cell free DNA (cfDNA). Circulating tumor DNA (ctDNA) which is cfDNA from tumor cells is shed into blood through apoptosis, necrosis or through an active process from neoplastic cells. Molecular aberrations in tumor tissues such as point mutations, insertions, deletions and methylation profiles are present in these circulating DNA fragments $(55,56,57,58,59)$. The fragmentation pattern of ctDNA in healthy people has been found to be different from the pattern in patients with various kinds of cancers (60). Surveillance circulating tumor DNA identifies patients at risk of recurrence in DLBCL with a positive predictive value of $88.2 \%$ and negative predictive value of $97.8 \%$ (61). Unlike CTCs and exosomes which contain RNA and proteins together with DNA, ctDNA provides no proteomic and transcriptomic information.

Immunoglobulin gene rearrangement is another novel technology for identifying prognostic groups in DLBC. Molecular characterization of immunoglobulin gene rearrangements revealed distinct subgroups in non-GCB (62). DLBCL patients with immunoglobulin gene rearrangement have been shown to have a lower rate of complete remission and significantly poorer survival. (63).

\section{Exosomes}

Exosomes are membrane-bound vesicles ranging from 30-120 nm in diameter, and are released upon fusion of multi-vesicular bodies with the cell membrane $(64,65,66)$. The contents of exosomes include proteins, nucleic acids, lipids and sugars, and they are thought to be involved in intercellular communication $(67,68,69,70,71)$. Exosomes are endosome derived extracellular vesicles. Early endosomes are formed from internal budding of the cell membrane with accompanying membrane proteins and lipids into the cytoplasm. As the endosome matures, there is transport of cytosolic, golgi and nuclear cargo such as nucleic acids, proteins and other metabolites into the lumen of the endosome. There is subsequently intravesicular budding of the endosomal membrane, forming multivesicular bodies (MVB). The MVB fuses with the cell membrane releasing its vesicles as exosomes into the extracellular space. Involvement of the endosomal pathway differentiates exosomes from microvesicles and apoptotic bodies which are the other extracellular vesicles. Microvesicles and apoptotic bodies are larger vesicles formed from outward budding of the cell membranes from living and dying cells respectively, into the extracellular space (72)

Mechanisms by which recipient cells take up exosomes include macropinocytosis, receptor or lipid raft mediated endocytosis, phagocytosis, or direct fusion with the recipient cell membrane (73). Exosomes are found in many body fluids including blood, urine, cerebrospinal fluid, amniotic fluid, saliva, breast milk, peritoneal fluid, etc. (74).

Exosome Isolation Methods 
There are various methods for isolating exosomes with varying degrees of complexity and contamination with non-exosomal vesicles and/or non-vesicular products. The prevalent method for exosome isolation is differential ultracentrifugation. It involves sequential spinning of a fluid at increasing speed and duration to pellet dead cells and cellular debris, microvesicles and eventually, exosomes (75). This method is not suitable for fluids with high viscosity such as blood since higher speeds and longer duration of centrifugation are required (76). Due to similarity of sedimentation properties of various types of extracellular vesicles the yield and purity using this method may be low $(77,78)$

Density gradient ultracentrifugation uses a concept similar to differential ultracentrifugation. The initial steps are similar to DUC but sucrose is added at a later point. Due to the buoyant density of exosomes (1.15 to $1.19 \mathrm{~g} / \mathrm{mL}$ ) in sucrose, it sediments separately from other denser extracellular vesicles during centrifugation $(79,80)$. This method yields purer fractions compared with differential ultracentrifugation (81), but has same disadvantage of need for long periods of processing and requirement of costly equipment.

Size based isolation techniques make use of the smaller size of exosomes compared with microvesicles, apoptotic bodies and other molecules to separate them in a fluid sample. Ultrafiltration, a size based technique uses membrane filters with decreasing pore sizes to isolate exosomes (82). It is a faster method compared with ultracentrifugation and does not require costly equipment. While this method yields pure vesicles, there is difficulty in removing contaminating proteins but can be combined with ultrcentrifugation for removal of proteins (83). Other size based techniques include size exclusion chromatography which involves differential passage of various vesicles and particles through a gel column and their recovery in different elution fractions (84). SEC allows for pure exosomal isolates with intact physical characteristics (85). The run time is however long and limits its use in multiple biological specimens.

In polymer-based precipitation, a polymer, e.g. polyethylene glycol (PEG) precipitates exosomes in a biofluid and the exosomes recovered by low speed centrifugation to pellet down the precipitate (86).

Immunoaffinity enrichment methods use exosomal membrane markers, typically the tetraspanins CD9, CD63 and CD81, to isolate exosomes from biofluids $(87,88)$. The antibodies are immobilized on varying media including magnetic beads, microfluidic devices and chromatography matrices $(89,90)$.

Newer methods of exosome isolation include contact-free sorting which uses ultrasound waves to exert differential acoustic forces on vesicles based on their size and density (91), microfluidics, which utilizes size dependent position of flow of various vesicles in a biofluid in a channel to isolate exosomes (92) and nanoplasmon-enhanced scatter which uses immobilized exosomal antibodies and gold plated nanoparticles to capture and detect exosomes based on scatter patterns (93). Confirmation of exosome isolation can be done using multiple techniques including electron microscopy which identifies the typical cup shaped morphology and vesicle size of 30-120nm, western blotting for exosome markers such as tetraspanins (CD63/CD9/CD81), ALIX, flotillin, hsp70, TSG101, and also nanoparticle tracking analysis for vesicle size and concentration determination (94).

\section{Exosome Use in DLBCL}

Due to their relative abundance, longer half-life, presence in multiple body fluids, and a payload that includes nucleic acids, proteins, and small molecules (95) exosomes are poised to contain biomarkers that may be informative in the prognostication and management of DLBCL patients at multiple stages. The nucleic acid and/or protein cargo in exosomes can be used for molecular analyses in DLBCL. Considering the relative abundance of exosomes in body fluids, especially serum where there is a concentration of about $3 \times 10^{6}$ exosomes/microliter, this presents a readily available source of biologic material for multiparametric studies leading to more exhaustive characterization of DLBCL types, prognostication, surveillance during and post treatment, and understanding of mechanisms of resistance to therapy. Rutherford et al characterized mutations in cell lines and their exosomes using RNA sequencing and found that nearly one third of mutations in the cell lines were detected in the exosomes (96). This was surprising as exosomes contained a smaller subset of RNAs than the cell lines, a finding that also suggests a likely enrichment of mutated RNA in exosomes. 


\section{Exosomes as Prognostic Markers in DLBCL}

Evaluation of exosomal nucleic acids can also provide additional prognostic information. Feng et al. found that circulating exosomal expression of miR-99a-5p and miR-125b-5p were significantly higher in chemoresistant vs. chemosensitive DLBCL patients $(\mathrm{p}<0.001)(97)$. Determination of predictive value of these miRNA using ROC curve analysis showed that the area under the curve (AUC) values of miR-99a-5p, miR-125b-5p, and IPI scores were $0.744(\mathrm{p}<0.001), 0.7802(\mathrm{p}<0.001)$, and $0.6308(\mathrm{p}<0.05)$, respectively. The combined predictive value of miR-99a-5p and IPI score, had AUC of $0.8326(\mathrm{p}<0.001)$ while that of miR-125b-5p combined with IPI score, had an AUC of $0.8143(\mathrm{p}<0.001)$. This suggests that determination of exosomal expression of miR-99a or miR-125b-5p, when combined with IPI may be a robust way to predict response to therapy. Zare et al. observed a significant increase in exosomal miR-155 levels in refractory/relapsed DLBCL patients compared to responding patients or patients still on R-CHOP therapy (98). They also observed a higher concentration in refractory/relapsed patients.

\section{Exosomes in Post Therapy Surveillance}

Exosomes and their associated cargo hold promise in their application as a non-invasive biomarker for DLBCL patients. They could be of great benefit in post treatment surveillance of DLBCL patients as a means to avoid radiation exposure of PET scans and unnecessary testing associated with false-positive PET scans. Di et al identified a panel of five circulating exosomal miRNAs as noninvasive biomarkers of DLBCL consisting of hsa-miR-379-5p, hsa-miR-135a-3p, hsa-miR-4476, hsa-miR-483-3p and hsa-miR-451a (99). hsamiR-379-5p, hsa-miR-135a-3p, hsa-miR-4476 were overexpressed in DLBCL compared to healthy controls, while hsa-miR-483-3p and hsa-miR-451a were expressed at lower levels. The panel had an AUC of 0.951 (95\% CI0.847-0.993) in the testing phase, 0.841 (95\% CI, 0.767-0.900) in the validation phase and 0.824 (95\% CI, 0.760-0.877) in the combined testing and validation phase.

\section{Exosomes as Mediators of Resistance and Targets for New Therapies}

Exosomes play crucial roles in survival of lymphoma cells, evasion of immune response and resistance to therapy. Poggio et al showed that exosomal PD-L1 suppressed T-cell function in lymph nodes draining tumor sites, and promoted tumor growth across different tumor types (100.). Local blockade of exosomal PD-L1, inhibited growth of tumor locally as well as at distant sites injected synchronously or at a later time to the PD-L1 block.

Tumor derived exosomes contain tumor antigens and MHC-I and MHC-II molecules allowing direct presentation and activation of CD8 and CD4 T-cells and cross presentation to T cells via transfer to antigen presenting cells $(101,102)$. Whether these mechanisms are also operational in DLBCL remains to be determined. Studies of exosomes have provided insights to mechanisms of resistance in DLBCL and possible targets for new therapy. Chen et al showed a dual role of tumor derived exosomes (TEX) in DLBCL (103.). DLBCL exosomes when incubated with dendritic cells (DC) resulted in increased proliferation and activation of dendritic cells. The dendritic cells were able to cause greater $\mathrm{T}$ cell expansion after incubation with the exosomes. Tumor derived exosomes however resulted in increased PD-1 expression and increased apoptosis of Th2 cells. They also demonstrated that the TEXs play a role in enhancing cell proliferation, invasion, migration, and angiogenesis with promotion of tumor growth in vivo. Their findings suggest a role for targeting exosome inhibition in developing new therapies for DLBCL or use of exosome derived vaccines to augment the anti-lymphoma immune response.

Koch et al demonstrated the presence of side population (SP) cells in DLBCL, which have stemness properties and are capable of propagating tumor growth (104). They showed an equilibrium of SP and non-SP cells, with no stemness properties, whereby exosome mediated Wnt signaling transformed non-SP cells to SP cells, and vice versa.

Exosomes have been shown to play a role in drug resistance of hematopoietic malignancies to therapy. Koch et al showed that after initial accumulation in the nucleus, the site of action of doxorubicin and pixantrone, these chemotherapeutic agents are exported into exosomes and then released from the cell, leading to reduced 
amount of drug at the site of action and development of resistance (105). They showed that inhibition of $\mathrm{ABCA} 3$, a protein involved in the transport of these drugs into exosomes resulted in trapping of the drugs in the nucleus and higher sensitivity to doxorubin and pixantrone.

Exosomal CD20 has also been shown to shield DLBCL cells from anti-CD20 immunotherapy providing a mechanism for evading this therapy (106). This mechanism of resistance was again attenuated by inhibition of ABCA3 which is involved in exosome biogenesis. Serial profiling of exosomal nucleic acids and/or proteins during therapy may identify more products involved in resistance and help develop a resistance signature during therapy that necessitates prompt therapy changes. It could also help guide development of new therapeutic targets.

\section{Challenges and Concluding Thoughts}

Since exosomes are released by multiple cell types, an obvious challenge in the use of exosomal cargo or liquid biopsy techniques in general as biomarkers of DLBCL is the enrichment of tumor specific exosomes in order to avoid dilution of information in tumor exosomes by exosomes from non-neoplastic tissue. Enrichment for tumor derived exosomes before subsequent analysis of exosomal cargo would address the issue of background noise from non-neoplastic exosomes. Castillo et al identified specific surface proteins on prostate cancer cell lines derived exosomes and used antibodies to these proteins for immunocapture in plasma of prostate cancer patients to enrich for tumor derived exosomes for downstream analysis of the tumor derived exosomal cargo (107). Mizutani et al also demonstrated isolation of prostate cancer specific exosomes using immunocapture with prostate specific antibodies. (108) Similar approaches can be employed as pre-analytical step in studying exosomes in fluids from DLBCL patients.

Another challenge in routine use of exosomes in cancer management is the difference in detected cargo observed using different methods of isolation. Jeppesen et al used high resolution gradient fractionation to separate small extracellular vesicles from non-vesicular proteins and direct immunoaffinity capture to isolate exosomes from other vesicles (109). Analyses of these exosomes showed absence in the cargo of some nucleic acids and proteins hitherto found to be present in exosomes using other methods. Contamination of exosomal isolates by other vesicles and non-vesicular molecules probably accounts for such variation. Guidelines to confirm purity of isolation would need to be developed to prevent inter-laboratory variation of exosomal content analyses.

Exosomes thus have great potential for not only improving diagnosis, prognostication, and monitoring of DLBCL, but provide a means of understanding the biology of DLBCL with the ultimate goal of improving care for patients.

\section{References}

1. Swerdlow SH, Campo E, Harris NL, Jaffe ES, Pileri SA, Stein H, Thiele J, Vardiman JW. WHO Classification of Tumours of Haematopoietic and Lymphoid Tissues. 4th ed. Lyon, France: IARC Press; 2008

2. Jaffe E.S.et al. Classification of lymphoid neoplasms: the microscope as a tool for disease discovery. Blood. 2008; 112(12):4384-99. doi: 10.1182/blood-2008-07-077982,

3. Laurini, J. A., Perry, A. M., Boilesen, E., Diebold, J., MacLennan, K. A., Müller-Hermelink, H. K., Nathwani, B. N., Armitage, J. O., \& Weisenburger, D. D.(2012). Classification of non-Hodgkin lymphoma in Central and South America: a review of 1028 cases. Blood, 120(24), 4795-4801. Accessed January 17, 2019. https://doi.org/10.1)

4. Teras, Lauren R., et al. "2016 US lymphoid malignancy statistics by World Health Organization subtypes." CA: a cancer journal for clinicians 66.6 (2016): 443-459

5. Morton, L. M., Wang, S. S., Devesa, S. S., Hartge, P., Weisenburger, D. D., \& Linet, M. S. (2006). Lymphoma incidence patterns by WHO subtype in the United States, 1992-2001. Blood, 107(1), 265276. Accessed January 17, 2019. https://doi.org/10.1182/blood-2005-06-2508

6. Shenoy, Pareen J., et al. "Racial differences in the presentation and outcomes of diffuse large B-cell lymphoma in the United States." Cancer 117.11 (2011): 2530-2540 
7. Howlader N, Noon AM, Krapcho M, et al., eds. SEER Cancer Statistics Review, 1975-2013, National Cancer Institute, Bethesda, MD, based on November 2015 SEER data submission, posted to the SEER web site, April 2016. https://seer.cancer.gov/csr/1975_2013/

8. Sant M, Minicozzi P, Mounier M, et al; EUROCARE-5 Working Group. Survival for haematological malignancies in Europe between 1997 and 2008 by region and age: results of EUROCARE-5, a population-based study. Lancet Oncol. 2014;15(9):931-942

9. Crump, M., Neelapu, S. S., Farooq, U., Van Den Neste, E., Kuruvilla, J., Westin, J., Gisselbrecht, C. (2017). Outcomes in refractory diffuse large B-cell lymphoma: results from the international SCHOLAR-1 study. Blood, 130(16), 1800-1808. doi:10.1182/blood-2017-03-769620

10. Lenz, G., Rutherford, S. C., Davies, A., Zinzani, P. L., Salles, G., Hasskarl, J., Margunato-Debay, S., Rodrigues, F., Nientker, L., Balradj, J., \& Alleman, C. The Burden of Relapsed/Refractory (R/R) Diffuse Large B-Cell Lymphoma (DLBCL): A Systematic Literature Review (SLR). Blood, 132(Suppl 1), 2241. Accessed January 17, 2019. https://doi.org/10.1182/blood-2018-99-112878.)

11. Friedberg, J.W. (2011) Relapsed/refractory diffuse large B-cell lymphoma. ASH Education Program Book, 2011, 498-505.)

12. Vose, J. M., Weisenburger, D. D., Loberiza, F. R., Arevalo, A., Bast, M., Armitage, J., .. \& Armitage, J. O. (2010). Late relapse in patients with diffuse large B-cell lymphoma. British journal of haematology, 151(4), 354-358

13. Broseus, J., Chen, G., Hergalant, S., Ramstein, G., Mounier, N., Gueant, J. L., .. Houlgatte, $R$. Relapsed diffuse large B-cell lymphoma present different genomic profiles between early and late relapses. Oncotarget, 7(51), 83987-84002. doi:10.18632/oncotarget.9793

14. Gisselbrecht $C$, Glass B, Mounier N, et al. Salvage regimens with autologous transplantation for relapsed large B-cell lymphoma in the rituximab era. J Clin Oncol. 2010;28(27):4184-4190, 10

15. R. Vaidya, T. E. Witzig, Prognostic factors for diffuse large B-cell lymphoma in the R(X)CHOP era, Annals of Oncology, Volume 25, Issue 11, November 2014, Pages 2124-2133, https://doi.org/10.1093/annonc/mdu109

16. Biccler, Jorne L., Sandra Eloranta, Peter de Nully Brown, Henrik Frederiksen, Mats Jerkeman, Judit Jorgensen, Lasse Hjort Jakobsen, Karin E. Smedby, Martin Bogsted, and Tarec C. El-Galaly. "Optimizing Outcome Prediction in Diffuse Large B-Cell Lymphoma by Use of Machine Learning and Nationwide Lymphoma Registries: A Nordic Lymphoma Group Study." JCO clinical cancer informatics 2 (2018): 1-13

17. International Non-Hodgkin's Lymphoma Prognostic Factors Project. (1993). A predictive model for aggressive non-Hodgkin's lymphoma. New England Journal of Medicine, 329(14), 987-994

18. Biccler, Jorne Lionel, Tarec Christoffer El-Galaly, Martin Bogsted, Judit Jorgensen, Peter de Nully Brown, Christian Bjorn Poulsen, Jorn Starklint et al. "Clinical prognostic scores are poor predictors of overall survival in various types of malignant lymphomas." Leukemia 83 lymphoma 60, no. 6 (2019): 1580-1583.

19. Sehn, L. H., Berry, B., Chhanabhai, M., Fitzgerald, C., Gill, K., Hoskins, P., .. \& \& Gascoyne, R. D. (2007). The revised International Prognostic Index (R-IPI) is a better predictor of outcome than the standard IPI for patients with diffuse large B-cell lymphoma treated with R-CHOP. Blood, 109(5), $1857-1861$

20. Alizadeh, A. A., Eisen, M. B., Davis, R. E., Ma, C., Lossos, I. S., Rosenwald, A., .. \& Powell, J. I. (2000). Distinct types of diffuse large B-cell lymphoma identified by gene expression profiling. Nature, 403(6769), 503

21. Christof Schneider, Laura Pasqualucci, Riccardo Dalla-Favera, Molecular pathogenesis of diffuse large B-cell lymphoma, Seminars in Diagnostic Pathology Volume 28, Issue 2,2011,Pages 167-177

22. Rosenwald A, Wright G, Chan WC, et al. The use of molecular profiling to predict survival after chemotherapy for diffuse large-B-cell lymphoma. N Engl J Med. 2002;346:1937-1947.

23. Vitolo, Umberto, et al. "Obinutuzumab or rituximab plus cyclophosphamide, doxorubicin, vincristine, and prednisone in previously untreated diffuse large B-cell lymphoma." Journal of Clinical Oncology 35.31 (2017): 3529-3537 
24. Scott DW, Mottok A, Ennishi D, et al. Prognostic Significance of Diffuse Large B-Cell Lymphoma Cell of Origin Determined by Digital Gene Expression in Formalin-Fixed Paraffin-Embedded Tissue Biopsies. J Clin Oncol. 2015;33(26):2848-2856. doi:10.1200/JCO.2014.60.2383

25. Xu-Monette, Zijun Y. et al "Mutational profile and prognostic significance of TP53 in diffuse large Bcell lymphoma patients treated with R-CHOP: report from an International DLBCL Rituximab-CHOP Consortium Program Study." Blood 120.19 (2012): 3986-3996. Web. 01 April. 2019.

26. Wilson, W. H., Young, R. M., Schmitz, R., Yang, Y., Pittaluga, S., Wright, G., .. \& De Vos, S. (2015). Targeting B cell receptor signaling with ibrutinib in diffuse large B cell lymphoma. Nature medicine, 21 (8), 922

27. Scott, D. W., Wright, G. W., Williams, P. M., Lih, C., Walsh, W., Jaffe, E. S., Rosenwald, A., Campo, E., Chan, W. C., Connors, J. M., Smeland, E. B., Mottok, A., Braziel, R. M., Ott, G., Delabie, J., Tubbs, R. R., Cook, J. R., Weisenburger, D. D., Greiner, T. C., Glinsmann-Gibson, B. J., Fu, K., Staudt, L. M., Gascoyne, R. D., \& Rimsza, L. M. (2014). Determining cell-of-origin subtypes of diffuse large B-cell lymphoma using gene expression in formalin-fixed paraffin-embedded tissue. Blood, 123(8), 1214-1217. Accessed July 07, 2019. https://doi.org/10.1182/blood-2013-11-536433.)

28. Hans, C. P., Weisenburger, D. D., Greiner, T. C., Gascoyne, R. D., Delabie, J., Ott, G., MullerHermelink, H. K., Campo, E., Braziel, R. M., Jaffe, E. S., Pan, Z., Farinha, P., Smith, L. M., Falini, B., Banham, A. H., Rosenwald, A., Staudt, L. M., Connors, J. M., Armitage, J. O., \& Chan, W. C. (2004). Confirmation of the molecular classification of diffuse large B-cell lymphoma by immunohistochemistry using a tissue microarray. Blood, 103(1), 275-282.Accessed April 10, 2019. https://doi.org/10.1182/blood-2003-05-1545

29. Visco C, Li Y, Xu-Monette ZY, et al. Comprehensive gene expression profiling and immunohistochemical studies support application of immunophenotypic algorithm for molecular subtype classification in diffuse large B-cell lymphoma: a report from the International DLBCL Rituximab-CHOP Consortium Program Study [published correction appears in Leukemia. 2014 Apr;28(4):980]. Leukemia. 2012;26(9):2103-2113. doi:10.1038/leu.2012.83

30. William W.L. Choi, Dennis D. Weisenburger, Timothy C. Greiner, Miguel A. Piris, Alison H. Banham, Jan Delabie, Rita M. Braziel, Huimin Geng, Javeed Iqbal, Georg Lenz, Julie M. Vose, Christine P. Hans, Kai Fu, Lynette M. Smith, Min Li, Zhongfeng Liu, Randy D. Gascoyne, Andreas Rosenwald, German Ott, Lisa M. Rimsza, Elias Campo, Elaine S. Jaffe, David L. Jaye, Louis M. Staudt and Wing C. Chan. A New Immunostain Algorithm Classifies Diffuse Large B-Cell Lymphoma into Molecular Subtypes with High Accuracy. Clin Cancer Res September 12009 (15) (17) 5494-5502; DOI: 10.1158/10780432.CCR-09-0113

31. Meyer PN, Fu K, Greiner TC, et al. Immunohistochemical methods for predicting cell of origin and survival in patients with diffuse large B-cell lymphoma treated with rituximab. J Clin Oncol. 2010;29(2):200-207. doi:10.1200/JCO.2010.30.036

32. Gutierrez-Garcia, G., Cardesa-Salzmann, T., Climent, F., Gonzalez-Barca, E., Mercadal, S., Mate, J. L., Sancho, J. M., Arenillas, L., Serrano, S., Escoda, L., Martinez, S., Valera, A., Martinez, A., Jares, P., Pinyol, M., Garcia-Herrera, A., Martinez-Trillos, A., Gine, E., Villamor, N., Campo, E., Colomo, L., Lopez-Guillermo, A., \& , . (2011) Blood, 117(18), 4836-4843. Accessed January 26, 2019. https://doi.org/10.1182/blood-2010-12-322362).

33. Yoon, Nara, et al. "Cell-of-origin of diffuse large B-cell lymphomas determined by the Lymph2Cx assay: better prognostic indicator than Hans algorithm." Oncotarget 8.13 (2017): 22014,

34. 25. Nyman H, Adde M, Karjalainen-Lindsberg ML, Taskinen M, Berglund M, Amini RM, Blomqvist C, Enblad G, Leppa S. Prognostic impact of immunohistochemically defined germinal center phenotype in diffuse large B-cell lymphoma patients treated with immunochemotherapy. Blood. 2007; 109:49304935.

35. Gleeson M, Hawkes EA, Cunningham D, Jack A, Linch D. Caution in the Use of Immunohistochemistry for Determination of Cell of Origin in Diffuse Large B-Cell Lymphoma. J Clin Oncol. 2015; 33:32153216

36. Schmitz, Roland, et al. "Genetics and pathogenesis of diffuse large B-cell lymphoma." New England 
Journal of Medicine378.15 (2018): 1396-1407

37. Chapuy, Bjoern, et al. "Molecular subtypes of diffuse large B cell lymphoma are associated with distinct pathogenic mechanisms and outcomes." Nature medicine 24.5 (2018): 679

38. Lopez, Juanita S., and Udai Banerji. "Combine and conquer: challenges for targeted therapy combinations in early phase trials." Nature reviews Clinical oncology 14.1 (2017): 57

39. Wierda, William G., et al. "NCCN guidelines insights: chronic lymphocytic leukemia/small lymphocytic lymphoma, version 1.2017." Journal of the National Comprehensive Cancer Network 15.3 (2017): 293-31

40. B.D. Cheson, R.I. Fisher, S.F. Barrington, et al.Recommendations for initial evaluation, staging, and response assessment of Hodgkin and non-Hodgkin lymphoma: the Lugano classification. J Clin Oncol, 32 (2014), pp. 3059-3068

41. Han HS, Escalon MP, Hsiao B, et al. High incidence of false-positive PET scans in patients with aggressive non-Hodgkin's lymphoma treated with rituximab-containing regimens. Ann Oncol 2009;20:309318.

42. 36. El-Galaly T, Prakash V, Christiansen I, et al. Efficacy of routine surveillance with positron emission tomography/computed tomography in aggressive non-Hodgkin lymphoma in complete remission: status in a single center. Leuk Lymphoma2011;52:597-60

43. Guppy AE, Tebbutt NC, Norman A, . The role of surveillance CT scans in patients with diffuse large B-cell non-Hodgkin's lymphoma. Leuk Lymphoma 2003;44:123-125

44. Cohen, J. B., Behera, M., Thompson, C. A., \& Flowers, C. R. (2017). Evaluating surveillance imaging for diffuse large B-cell lymphoma and Hodgkin lymphoma. Blood, 129(5), 561-564. Accessed February 02, 2019.https://doi.org/10.1182/blood-2016-08-685073

45. Durani, U., Asante, D., Heien, H. C., Thompson, C. A., Halfdanarson, T. R., Peethambaram, P., Quevedo, J. F., Villasboas, J. C., \& Go, R. S. (2018). Changes in Imaging Surveillance of Diffuse Large B-Cell Lymphoma Survivors after Publication of the American Society of Hematology Choosing Wisely(r)Recommendations. Blood, 132(Suppl 1), 618. Accessed February 04, 2019.https://doi.org/10.1182/blood-2018-99-119179

46. Smith-Bindman R, Lipson J, Marcus R. Radiation dose associated with common computed tomography examinations and the associated lifetime attributable risk of cancer. Arch Intern Med 2009; 169:20782086

47. Alix-Panabieres C, Pantel K. Circulating tumor cells: liquid biopsy of cancer. Clin Chem. 2013 Jan; $59(1): 110-8$

48. Krebs, M. G. et al. Molecular analysis of circulating tumour cells - biology and biomarkers. Nat. Rev. Clin. Oncol. 11, 129-144 (2014

49. Siravegna G, Marsoni S, Siena S, Bardelli A. Integrating liquid biopsies into the management of cancer. Nat Rev Clin Oncol. 2017 Sep;14(9):531-548. doi: 10.1038/nrclinonc.2017.14. Epub 2017 Mar 2

50. Haber, D. A. \& Velculescu, V. E. Blood-based analyses of cancer: circulating tumor cells and circulating tumor DNA. Cancer Discov. 4, 650-661 (2014

51. Riethdorf S, Fritsche H, Muller V, Rau T, Schindlbeck C, Rack B, et al. Detection of circulating tumor cells in peripheral blood of patients with metastatic breast cancer:a validation study of the CellSearch system. Clin Cancer Res. 2007;13:920-8

52. Talasaz AH, Powell AA, Huber DE, Berbee JG, Roh KH, Yu W, et al. Isolating highly enriched populations of circulating epithelial cells and other rare cells from blood using a magnetic sweeper device. Proc Natl Acad Sci U S A. 2009;106:3970-5

53. Nagrath S, Sequist LV, Maheswaran S, Bell DW, Irimia D, Ulkus L, et al. Isolation of rare circulating tumour cells in cancer patients by microchip technology. Nature. 2007;450:1235-9

54. Brock, G., Castellanos-Rizaldos, E., Hu, L., Coticchia, C., \& Skog, J. (2015). Liquid biopsy for cancer screening, patient stratification and monitoring. Translational Cancer Research, 4(3), 280-290

55. Bettegowda, C. et al. Detection of circulating tumor DNA in early- and late-stage human malignancies.Sci. Transl Med. 6, $224 \mathrm{ra} 24$ (2014

56. Thierry, A. R. et al. Clinical validation of the detection of KRAS and BRAF mutations from circulating 
tumor DNA. Nat. Med. 20, 430-435 (2014

57. Narayan, A. et al. Ultrasensitive measurement of hotspot mutations in tumor DNA in blood using error- suppressed multiplexed deep sequencing. Cancer Res. 72, 3492-3498 (2012),

58. Liggett, T. et al. Differential methylation of cell-free circulating DNA among patients with pancreatic cancer versus chronic pancreatitis. Cancer 116, 1674-1680 (2010),

59. Sturgeon, S. R. et al. Detection of promoter methylation of tumor suppressor genes in serum DNA of breast cancer cases and benign breast disease controls. Epigenetics 7, 1258-1267 (2012)

60. Cristiano, Stephen, Alessandro Leal, Jillian Phallen, Jacob Fiksel, Vilmos Adleff, Daniel C. Bruhm, Sarah Ostrup Jensen et al. "Genome-wide cell-free DNA fragmentation in patients with cancer." Nature (2019): 1.

61. Roschewski M, Dunleavy K, Pittaluga S, et al. Circulating tumour DNA and CT monitoring in 124patients with untreated diffuse large B-cell lymphoma: a correlative biomarker study. Lancet 125Oncol. 2015;16(5):541-549.1264.

62. Sebastian, E., Alcoceba, M., Balanzategui, A., Marin, L., Montes-Moreno, S., Flores, T., ... \& Corral, R. (2012). Molecular characterization of immunoglobulin gene rearrangements in diffuse large B-cell lymphoma: antigen-driven origin and IGHV4-34 as a particular subgroup of the non-GCB subtype. The American journal of pathology , 181 (5), 1879-1888.

63. Mitterbauer-Hohendanner, G., Mannhalter, C., Winkler, K., Mitterbauer, M., Skrabs, C., Chott, A., .. \& \& Jaeger, U. (2004). Prognostic significance of molecular staging by PCR-amplification of immunoglobulin gene rearrangements in diffuse large B-cell lymphoma (DLBCL). Leukemia , 18 (6), 1102.

64. Hurley JH, Odorizzi G. Get on the exosome bus with ALIX. Nat Cell Biol. 2012; 14:654-655. https://doi.org/10.1038/ncb2530

65. Zhang X, Yuan X, Shi H, Wu L, Qian H, Xu W. Exosomes in cancer: small particle, big player. J. Hematol. Oncol. 8, 83 (2015)

66. Fevrier B, Raposo G. Exosomes: endosomal-derived vesicles shipping extracellular messages. Curr Opin Cell Biol 2004;16(4):415-421

67. Mathieu, M., Martin-Jaular, L., Lavieu, G., \& Thery, C. (2019). Specificities of secretion and uptake of exosomes and other extracellular vesicles for cell-to-cell communication. Nature cell biology, 21(1), 9.

68. C. Lasser, M. Eldh, J. Lotvall Isolation and characterization of RNA-containing exosomes J. Vis. Exp., 59 (2012), pp. 3037-3047,

69. Valadi H, Ekstrom K, Bossios A, Sjostrand M, Lee JJ, Lotvall JO. Exosome-mediated transfer of mRNAs and microRNAs is a novel mechanism of genetic exchange between cells. Nat. Cell Biol. 9(6), 654-659 (2007), C.

70. Subra, K. Laulagnier, B. Perret, M. Record. Exosome lipidomics unravels lipid sorting at the level of multivesicular bodies Biochimie, 89 (2007), pp. 205-212,

71. B.S. Batista, W.S. Eng, K.T. Pilobello, K.D. Hendricks-Munoz, L.K. Mahal Identification of a conserved glycan signature for microvesicles. J. Proteome Res., 10 (2011), pp. 4624-4633

72. Crescitelli, Rossella, Cecilia Lasser, Tamas G. Szabo, Agnes Kittel, Maria Eldh, Irma Dianzani, Edit I. Buzas, and Jan Lotvall. "Distinct RNA profiles in subpopulations of extracellular vesicles: apoptotic bodies, microvesicles and exosomes." Journal of extracellular vesicles 2, no. 1 (2013): 20677

73. Jeffrey S. Schorey and Sanchita Bhatnagar1. Exosome Function: From Tumor Immunology to Pathogen Biology. Traffic 2008; 9:871-881Blackwell Munksgaard

74. Xia, Bing, et al. "The central roles of exosomes in hematological malignancies: A new frontier review." Bioscience Biotechnology Research Comminications11.3 (2018): 347-355

75. Mikhail A. Livshits, Elena Khomyakova, Evgeniy G. Evtushenko, Vassili N. Lazarev, Nikolay A. Kulemin, Svetlana E. Semina, Edward V. Generozov \& Vadim M. Govorun. Isolation of exosomes by differential centrifugation: Theoretical analysis of a commonly used protocol Scientific Reports volume 5, Article number: 17319 (2015

76. Momen Heravi F, Balaj L, Alian S, Trachtenberg A, Hochberg F, Skog J, et al. Impact of biofluid 
viscosity on size and sedimentation efficiency of the isolated microvesicles. Front Physiol. 2012;3:162

77. Lane, R. E., Korbie, D., Anderson, W., Vaidyanathan, R. \& Trau, M. Analysis of exosome purification methods using a model liposome system and tunable-resistive pulse sensing. Sci Rep. 5, 7639 (2015)

78. Witwer, K. W. et al. Standardization of sample collection, isolation and analysis methods in extracellular vesicle research. J Extracell Vesicles. 2, 20360 (2013)

79. Thery, C.; Amigorena, S.; Raposo, G.; Clayton, A. Isolation and Characterization of Exosomes From Cell Culture Supernatants and Biological Fluids. Current Protocols in Cell Biology: Hoboken, NJ, 2006; Chapter 3, Unit 3.22, DOI: 10.1002/0471143030.cb0322s30,

80. Vidal, M.; Mangeat, P.; Hoekstra, D. Aggregation Reroutes Molecules From a Recycling to a VesicleMediated Secretion Pathway During Reticulocyte Maturation J. Cell Sci. 1997, 110, 1867- 1877

81. Huilin Shao, Hyungsoon Im, Cesar M. Castro, Xandra Breakefield, Ralph Weissleder, and Hakho Lee. New Technologies for Analysis of Extracellular Vesicles. Chemical Reviews 2018118 (4), 1917-1950 DOI: 10.1021 /acs.chemrev.7b00534

82. Heinemann, M. L., Ilmer, M., Silva, L. P., Hawke, D. H., Recio, A., Vorontsova, M. A., ... \& Vykoukal, J. (2014). Benchtop isolation and characterization of functional exosomes by sequential filtration. Journal of Chromatography A, 1371, 125-135.

83. Alvarez, M.L.; Khosroheidari, M.; Kanchi Ravi, R.; DiStefano, J.K. Comparison of protein, microRNA, and mRNA yields using different methods of urinary exosome isolation for the discovery of kidney disease biomarkers. Kidney Int. 2012, 82, 1024-1032

84. Gamez-Valero, A., Monguio-Tortajada, M., Carreras-Planella, L., Beyer, K., \& Borras, F. E. (2016). Size-Exclusion Chromatography-based isolation minimally alters Extracellular Vesicles' characteristics compared to precipitating agents. Scientific reports, 6, 33641.

85. Taylor D, Zacharias W, Gercel Taylor C. Exosome isolation for proteomic analyses and RNA profiling. Methods Mol Biol. 2011; 728:235-46

86. Taylor, D. D., \& Shah, S. (2015). Methods of isolating extracellular vesicles impact down-stream analyses of their cargoes. Methods, 87, 3-10). The disadvantage of this method is significant coisolation with non-exosomal molecules and limited suitability of recovered exosomes for downstream analyses.

87. B. J. Tauro, D. W. Greening, R. A. Mathias et al., "Comparison of ultracentrifugation, density gradient separation, and immunoaffinity capture methods for isolating human colon cancer cell line LIM1863derived exosomes," Methods, vol. 56, no. 2, pp. 293-304, 2012.,

88. K. A. Konadu, M. B. Huang, W. Roth et al., "Isolation of exosomes from the plasma of HIV-1 positive individuals," Journal of Visualized Experiments, vol. 2016, no. 107, Article ID e53495, 2016

89. Zeringer, E., Barta, T., Li, M., \& Vlassov, A. V. (2015). Strategies for isolation of exosomes. Cold Spring Harbor Protocols, 2015(4), pdb-top074476). Immunoaffinity methods yield isolates of high purity but have low capacity.

90. Witwer, K. W., Buzas, E. I., Bemis, L. T., Bora, A., Lasser, C., \& Lotvall, J. (2013). Nolte-'t Hoen EN, Piper MG, Sivaraman S, Skog J, Thery C, Wauben MH, Hochberg F. Standardization of sample collection, isolation and analysis methods in extracellular vesicle research. J Extracell Vesicles, $2(10.3402$

91. Lee, K.; Shao, H.; Weissleder, R.; Lee, H. Acoustic Purification of Extracellular Microvesicles ACS Nano 2015, 9, 2321- 2327 DOI: 10.1021/nn506538f

92. Liu, C., Guo, J., Tian, F., Yang, N., Yan, F., Ding, Y., .. \& Sun, J. (2017). Field-free isolation of exosomes from extracellular vesicles by microfluidic viscoelastic flows. ACS nano, 11(7), 6968-6976

93. R. C. Lai, R. W. Y. Yeo, K. H. Tan, and S. K. Lim, "Exosomes for drug delivery - a novel application for the mesenchymal stem cell," Biotechnology Advances, vol. 31, no. 5, pp. 543-551, 2013

94. Vlassov, A. V., Magdaleno, S., Setterquist, R., \& Conrad, R. (2012). Exosomes: current knowledge of their composition, biological functions, and diagnostic and therapeutic potentials. Biochimica et Biophysica Acta (BBA)-General Subjects, 1820(7), 940-948

95. Alimirzaie, Sahar, Maryam Bagherzadeh, and Mohammad R. Akbari. "Liquid Biopsy in Breast Cancer: A Comprehensive Review." Clinical genetics (2019 
96. Rutherford, Sarah C., Angela Fachel, Sheng Li, Yanwen Jiang, Maria Pilar Dominguez, Doron Betel, and Rita Shaknovich. "DLBCL-Derived Exosomes Provide Key Insights into Genomic Landscape in Cell of Origin and May Lead to a Novel Method of Surveillance and Therapeutic Intervention." (2017): 5132-5132.

97. Feng, Yuhua, Meizuo Zhong, Shan Zeng, Leyuan Wang, Ping Liu, Xiangyu Xiao, and Yiping Liu. "Exosome-derived miRNAs as predictive biomarkers for diffuse large B-cell lymphoma chemotherapy resistance." Epigenomics 11, no. 1 (2018): 35-51

98. Nasrin Zare, Shaghayegh Haghjooy Javanmard, Valiollah Mehrzad, Nahid Eskandari \& Amirhosein Kefayat (2019) Evaluation of exosomal miR-155, let-7g and let-7i levels as a potential noninvasive biomarker among refractory/relapsed patients, responsive patients and patients receiving R-CHOP, Leukemia \& Lymphoma, DOI: 10.1080/10428194.2018.1563692

99. Di, C., Jiang, Y., Li, M., Juan, X., \& Xu, C. (2018). Circulating Exosomal microRNA Signature As a Noninvasive Biomarker for Diagnosis of Diffuse Large B-Cell Lymphoma. Blood, 132(Suppl 1), 5406. Accessed March 10, 2019. https://doi.org/10.1182/blood-2018-99-115940

100. Poggio M, Hu T, Pai CC, Chu B, Belair CD, Chang A, Montabana E, Lang UE, Fu Q, Fong L, Blelloch R. Suppression of Exosomal PD-L1 Induces Systemic Anti-Tumor Immunity and Memory. Cell. 2019 Apr 4;177(2):414-427.e13. doi: 10.1016/j.cell.2019.02.016

101. G Raposo, H W Nijman, W Stoorvogel, R Liejendekker, C V Harding, C J Melief, H J Geuze. B lymphocytes secrete antigen-presenting vesicles. Journal of Experimental Medicine Mar 1996, 183 (3) 1161-1172; DOI: 10.1084/jem.183.3.1161

102. 61. Chaput, Nathalie, and Clotilde Thery. "Exosomes: immune properties and potential clinical implementations." In Seminars in immunopathology, vol. 33, no. 5, pp. 419-440. Springer-Verlag, 2011.

103. Chen, Zhenzhen, Liangshun You, Lei Wang, Xianbo Huang, Hui Liu, Ju ying Wei, Li Zhu, and Wenbin Qian. "Dual effect of DLBCL-derived EXOs in lymphoma to improve DC vaccine efficacy in vitro while favor tumorgenesis in vivo." Journal of Experimental \& Clinical Cancer Research 37, no. 1 (2018): 190

104. Koch, Raphael, et al. "Populational equilibrium through exosome-mediated Wnt signaling in tumor progression of diffuse large B-cell lymphoma." Blood 123.14 (2014): 2189-2198.

105. Koch, R., Aung, T., Vogel, D., Chapuy, B., Wenzel, D., Becker, S., .. \& Truemper, L. (2016). Nuclear trapping through inhibition of exosomal export by indomethacin increases cytostatic efficacy of doxorubicin and pixantrone. Clinical cancer research, 22(2), 395-404

106. Aung, Thiha, et al. "Exosomal evasion of humoral immunotherapy in aggressive B-cell lymphoma modulated by ATP-binding cassette transporter A3." Proceedings of the National Academy of Sciences 108.37 (2011): 15336-15341

107. J Castillo, V Bernard, F A San Lucas, K Allenson, M Capello, D U Kim, P Gascoyne, F C Mulu, B M Stephens, J Huang, H Wang, A A Momin, R O Jacamo, M Katz, R Wolff, M Javle, G Varadhachary, I I Wistuba, S Hanash, A Maitra, H Alvarez, Surfaceome profiling enables isolation of cancer-specific exosomal cargo in liquid biopsies from pancreatic cancer patients, Annals of Oncology, Volume 29, Issue 1, January 2018, Pages 223-229, https://doi.org/10.1093/annonc/mdx542

108. Mizutani, Kosuke, Riyako Terazawa, Koji Kameyama, Taku Kato, Kengo Horie, Tomohiro Tsuchiya, Kensaku Seike et al. "Isolation of prostate cancer-related exosomes." Anticancer research 34, no. 7 (2014): 3419-3423.

109. Jeppesen, Dennis K., Aidan M. Fenix, Jeffrey L. Franklin, James N. Higginbotham, Qin Zhang, Lisa J. Zimmerman, Daniel C. Liebler et al. "Reassessment of exosome composition." Cell 177, no. 2 (2019): $428-445$

\section{Hosted file}

Ofori et al-Figures.pptx available at https://authorea.com/users/325867/articles/453873exosomes-as-liquid-biopsy-biomarkers-in-diffuse-large-b-cell-lymphoma-dlbcl-currentstate-of-the-art-and-unmet-clinical-needs 